\title{
LA PolynéSIE FRANÇAISE ET L'EURO
}

\author{
Vincent Dropsy*
}

The legal status of French Polynesia has important implications for the local economy, in particular for its currency, the CFP franc. A resolution passed on January 19, 2006 by the Assembly of French Polynesia shows the political will to replace this currency by the euro. This article explains the institutional procedures necessary for a possible switch to the euro in French Polynesia, and analyses its economic advantages and drawbacks.

The conclusion is that entering the euro zone would be in the best interests of French Polynesia.

Le statut juridique de la Polynésie française a d'importantes conséquences sur l'économie locale, en particulier sur sa monnaie, le franc CFP. Une résolution adoptée le 19 janvier 2006 par l'Assemblée de la Polynésie française montre la volonté politique actuelle de remplacer cette monnaie par l'euro. Cet article présente les modalités institutionnelles d'un éventuel passage à l'euro en Polynésie française, et analyse ses avantages et inconvénients d'un point de vue économique.

\section{INTRODUCTION}

La récente résolution de l'Assemblée de la Polynésie française en vue de remplacer le franc CFP par l'euro a soulevé de nombreuses questions à cause du statut juridique particulier de la Polynésie française.

Premièrement, du point du vue institutionnel, les lois statutaires du 6 septembre 1984, puis du 27 février 2004 confèrent à la Polynésie française une autonomie grandissante, mais le privilège d'émission de la monnaie locale est toujours sous le contrôle de la Banque de France, à travers son Institut d'Émission d'Outre-Mer (IEOM). La procédure juridique pour obtenir l'autorisation de

* Professeur d'économie internationale à California State University, Fullerton. Je tiens à exprimer ma gratitude aux professeurs Bernard Poirine et Christian Montet pour leur précieuse collaboration et leur accueil chaleureux, ainsi qu'au Ministère de l'Outre-Mer et à l'Université de la Polynésie Française pour leur soutien de ce projet de recherche. Je tiens également à remercier les économistes de Polynésie Française et en particulier Louis Savoie, Julien Vucher-Visin, Olivier Champion, Benoît Massuyeau, Jean-Pierre Derancourt, Frédéric Donzel, John Tehuritaua, Nicolas Brun et bien d'autres dans les services publics locaux et à l'université pour leur généreuse aide. Version complétée et révisée d'une présentation donnée le 24 mai 2006 lors du cycle de conférence «savoir pour tous» à l'Université de la Polynésie Française (UPF). Cet article est le fruit d'une recherche de l'auteur pendant son année sabbatique à l'UPF. 
passer à l'euro en Polynésie française est loin d'être simple, puisqu'elle comprend de nombreuses modalités au niveau de l'Union Européenne (UE) et de la Banque Centrale Européenne (BCE): 26 directives, 6 règlements, 9 décisions du Conseil Européen, 3 recommandations de la Commission Européenne et 5 orientations de la BCE. Auparavant, il faut également obtenir l'accord de la Nouvelle Calédonie et de Wallis et Futuna, ce qui n'est pas encore le cas en 2006, ainsi que du gouvernement français à travers la Banque de France. Ceci explique pourquoi il existe un délai incompressible est de trois ans entre cet accord et l'introduction éventuelle de l'euro en Polynésie française.

Deuxièmement, du point de vue économique, la Banque de France maintient une parité fixe depuis 1949 entre le franc CFP (F CFP) et le franc français (FF) et depuis le $1^{\text {er }}$ janvier 1999, entre le franc CFP et l'euro $(€)$. Elle assure également une convertibilité illimitée pour le franc CFP, à travers un compte d'opérations ouvert au nom de l'IEOM dans les livres du Trésor Public de la France. Cela signifie que la Polynésie française est libérée de toute contrainte économique externe au niveau de la défense de la parité de sa monnaie. En d'autres termes, les résidents de Polynésie française (dans le domaine privé ou public) peuvent continuer à importer maintes fois plus qu'ils n'exportent sans se soucier des conséquences macroéconomiques d'un tel déficit commercial, c'est-àdire une éventuelle dévaluation de la monnaie locale. Tant que l'Etat français continuera à financer ces déficits externes privés par des transferts publics du même ordre (environ 30\% du produit intérieur brut), le passage à l'euro ne changera pas cette situation très favorable à la Polynésie française. Par contre, si ces transferts publics faiblissent nettement, l'économie polynésienne pourrait en souffrir gravement. Il faut donc considérer les coûts macroéconomiques possibles dus à l'appartenance à l'Union Monétaire Européenne (UME) dans ce cas.

En tout cas, le statut juridique particulier de la Polynésie française lui éviterait d'avoir à satisfaire les critères dits de Maastricht, du nom du traité précédant l'UME. Puisque la Polynésie française est partie intégrante de la France, elle appartient au groupe des "Pays et Territoires d'Outre-Mer" (PTOM) pour employer le jargon européen. Sa politique monétaire est déterminée par la Banque de France et la France est seule à avoir la responsabilité de satisfaire des critères d'appartenance à l'UME, tels qu'un déficit budgétaire inférieur à 3\% du Produit Intérieur Brut (PIB) ou une dette gouvernementale inférieure à $60 \%$ du PIB. En d'autres termes, cela signifie que la Polynésie française reste totalement libérée des contraintes européennes même si l'euro devenait sa monnaie. De plus, les rapports de l'Assemblée de la Polynésie française, ${ }^{1}$ du Conseil Economique et Social et Culturel de la Polynésie française (CESC), ${ }^{2}$ de la Chambre De Commerce, d'Industrie, des

1 Assemblée de la Polynésie Française, Commission des Finances (19 janvier 2006), "Rapport concernant une proposition de résolution relative à l'introduction de l'euro en Polynésie Française", n 011-2006.

2 Conseil Economique et Social et Culturel de la Polynésie Française (15 novembre 2005) "Projet d'avis relatif au remplacement du franc CFP par l'euro en Polynésie Française" (Saisine du Gouvernement). 
Services et des Métiers de Polynésie française (CCISM), ${ }^{3}$ et d'autres excellents articles ${ }^{4}$ montrent clairement que l'introduction de l'euro présente des avantages microéconomiques non négligeables par rapport au régime monétaire actuel du franc CFP. Cela ne signifie pas nécessairement que l'adoption de l'euro en tant que monnaie locale correspond au choix d'un régime de change optimal. Il existe évidemment d'autres monnaies que l'euro, tels que le dollar U.S., et d'autres régimes de change possibles. Une analyse de l'économie polynésienne révèlera que l'euro est clairement le choix optimal pour ancrer la monnaie locale et que le remplacement du franc CFP par l'euro est également le meilleur choix dans le contexte actuel. En particulier, il faudra discuter des difficultés économiques dues à la surévaluation de la monnaie locale et de l'impact du passage à l'euro.

Cet article a donc pour but de présenter les modalités et contraintes institutionnelles d'un éventuel passage à l'euro en Polynésie française dans un deuxième chapitre, et d'analyser ses avantages et inconvénients d'un point de vue économique dans un troisième chapitre, avant de montrer la nécessité grandissante de réduire la dépendance de l'économie polynésienne vis-à-vis des transferts financiers de l'Etat français en conclusion.

\section{MODALITÉS ET CONTRAINTES INSTITUTIONNELLES DU PASSAGE À L'EURO}

\section{A Historique du franc CFP en Polynésie Française}

Depuis le 25 décembre 1945, la monnaie légale de Polynésie française est le franc CFP (franc des Comptoirs Français du Pacifique), comme dans les autres "Territoires d'Outre-Mer"5 (la Nouvelle Calédonie ainsi que Wallis et Futuna, anciennement appelés Nouvelles-Hébrides). Elle remplace le franc français, qui était en circulation légale depuis le 20 novembre 1843 (à la suite du traité de protectorat du 25 mars), tout en coexistant avec d'autres monnaies en parallèle, comme le dollar pendant la seconde guerre mondiale. Avant 1843, diverses monnaies étaient utilisées, telles que les piastres d'Amérique du Sud, mais le troc avec les navigateurs et la générosité polynésienne étaient également à la base des échanges. ${ }^{6}$

Pendant la deuxième guerre mondiale, la présence de troupes américaines à Bora Bora et l'absence d'échanges avec la métropole avait conféré de facto au dollar US (\$) le rôle de monnaie

3 Chambre de Commerce, d'Industrie, des Services et des Métiers de Polynésie Française (2005) "Note sur l'euro" par Benjamin Angel, John Tehuritaua, Maire Liu.

4 Louis Savoie (octobre 2006) "La Polynésie a voté pour l'Euro ?", DIXIT, $\mathrm{n}^{\circ} 15$. Louis Savoie (octobre $2005)$ "L'Euro en Polynésie Française, quelles obligations? Quels avantages?" DIXIT, n¹4, 112-116. Louis Savoie (septembre 2005) "Enjeux de l'introduction de l'euro à "Tahiti Nui" Tahiti Pacifique n¹73, 33-35.

5 Depuis la loi statutaire du 27 février 2004, la Polynésie Française est devenue une "Collectivité d'OutreMer" (COM). Depuis la loi organique du 19 mars 1999, la Nouvelle Calédonie est également devenu une COM, ainsi que Wallis et Futuna depuis la révision constitutionnelle du 28 mars 2003.

6 Christian Beslu Tahiti et sa monnaie (Pacific Promotion, Tahiti, 1997). 
locale, ${ }^{7}$ de sorte que le taux de change du franc CFP a été fixé le 25 décembre 1945 par rapport au dollar ( $1 \$=50 \mathrm{~F}$ CFP) plutôt qu'au franc français ( $1 \mathrm{~F} \mathrm{CFP}=2,40 \mathrm{FF})$. Jusqu'au $1^{\text {er }}$ septembre 1949, ce taux fixe F CFP/\$ a été maintenue alors que le franc métropolitain subissait des dévaluations successives. Depuis cette date, le taux de change du franc CFP est fixe par rapport au franc français, et non plus au dollar. Plus précisément, la valeur de 1000 francs CFP a été maintenu à une parité fixe de 5500 anciens francs avant 1958, date à laquelle l'ancien franc a été remplacé par le nouveau franc (FF), d'où un taux de change de $55 \mathrm{FF}$. Au $1^{\text {er }}$ janvier 1999, d'abord dans les marchés financiers, puis au $1^{\text {er }}$ janvier 2002, dans tous les autres marchés monétaires (billets et pièces), le franc français a été substitué par l'euro en métropole ainsi que dans les Départements d'Outre-Mer (DOM), à Mayotte, et à Saint Pierre et Miquelon, mais pas en Polynésie française ni en Nouvelle Calédonie ou à Wallis et Futuna, où la valeur de 1000 francs CFP est égale à exactement 8,38 euros (approximativement 1 euro $=119,33 \mathrm{~F} \mathrm{CFP}$ ).

La mise en circulation des francs CFP est la responsabilité de l'Institut d'Émission d'Outre-Mer (IEOM) depuis le 30 mars 1967, même si les billets et pièces sont fabriqués par la Banque de France. Ces privilèges étaient auparavant réservés à la Banque d'Indochine depuis 1888. Il faut également souligner que l'IEOM «procède au refinancement des établissements de crédit au moyen du réescompte des crédits bancaires consentis aux entreprises des secteurs considérés comme prioritaires et qui présentent une situation financière équilibrée». ${ }^{8}$ Elle veille aussi au bon fonctionnement du système bancaire local. Depuis 1970, l'IEOM présente un bilan économique, monétaire et financier de la Polynésie française, dans un rapport annuel considéré comme une référence en la matière. Depuis 1998, en conjonction avec la Banque de France, l'IEOM calcule également une balance des paiements ${ }^{9}$ de la Polynésie française, qui fournit des informations précieuses sur les échanges avec l'extérieur (flux représentant opérations sur biens et services, revenus, transferts courants et flux financiers).

Elle assure aussi la convertibilité illimitée du franc CFP en euro (auparavant en franc français) à travers un compte d'opérations ouvert dans les livres du Trésor public. Cependant, il faut savoir que l'IEOM ne détient pas de réserves internationales et c'est la Banque de France qui gère les réserves de change ainsi que la fixité du taux de change entre le franc CFP et l'euro. On verra que ce système monétaire particulier a d'importantes conséquences économiques pour Polynésie française.

7 Le mot tahitien "tara" pour désigner une unité monétaire, encore aujourd'hui dans certaines îles, est un dérivé phonétique du mot "dollar".

8 Institut d'Emission d'Outre-Mer (2006) "La Polynésie Française en 2005", Rapport Annuel.

9 Institut d'Emission d'Outre-Mer (2006) "La Balance des Paiements de la Polynésie Française en 2005", Rapport Annuel. 


\section{B Modalités Institutionnelles du Passage à l'euro}

Si la France garde «le privilège d'émettre des monnaies dans ses territoires d'outre-mer selon les modalités établies par sa législation nationale» et reste «seule habilitée à déterminer la parité du franc CFP» selon le protocole $\mathrm{n}^{\circ} 13 \mathrm{du}$ traité de Maastricht sur l'Union Européenne (UE), le gouvernement français actuel a clairement indiqué par la voix de son ministre de l'outre-mer que le passage à l'euro ne serait possible que si ce changement de monnaie ne se ferait que simultanément et qu'avec l'accord formel des autorités des trois collectivités d'outremer du Pacifique. Puisque l'Assemblée de la Polynésie française a adopté une résolution en faveur de l'introduction de l'euro en remplacement du franc CFP le 19 janvier 2006, il reste à la Nouvelle Calédonie et à Wallis et Futuna à s'exprimer formellement sur ce sujet avant qu'une demande à l'Etat (par lettre au Ministre de l'Économie et des Finances) soit faite par les trois collectivités d'outre-mer. Le gouvernement français devrait en principe donner son accord au passage à l'euro, puisqu'il y semble favorable, selon les commentaires recueillis du Président de la République. Une fois l'accord obtenu au niveau européen, l'Etat devrait informer le gouvernement de la Polynésie française en application de l'article 100 de la loi statutaire de 2004 avant de remplacer le franc CFP par l'euro.

Les modalités institutionnelles au niveau européen sont en revanche plus compliquées et plus longues, même si elles n'apparaissent pas comme un obstacle majeur. ${ }^{10}$ En effet, deux spécialistes de Bruxelles, ${ }^{11}$ qui sont venus à Tahiti en septembre 2005 pour informer les autorités et entreprises locales sur les modalités et enjeux du passage à l'euro en Polynésie française, ont déclaré qu'il n'était pas nécessaire de modifier le traité de Maastricht, ce qui aurait nécessité la ratification des 25 États membres. Il suffirait que le Ministre de l'Economie et des Finances de la France transmette au Commissaire Européen chargé des Affaires Économiques et Monétaires une demande de renonciation du protocole $n^{\circ} 13$ et d'ouverture de négociations en vue d'une introduction de l'euro dans les trois collectivités d'outremer du Pacifique, considérés comme des Pays et Territoires d'Outre-Mer (PTOM) par l'UE. ${ }^{12}$ Ensuite, la Commission Européenne enverrait une recommandation au Conseil de l'UE en vue de l'ouverture des négociations monétaires avec la France. La Banque Centrale Européenne (BCE) donnerait son avis sur cette recommandation. Le Conseil de l'UE déciderait l'ouverture des négociations et donnerait à la Commission Européenne un mandat de négociation pour trouver un accord monétaire avec la France en vue d'introduire l'euro

10 Source: Chambre De Commerce, d'Industrie, des Services et des Métiers de Polynésie Française (2005), "Note sur l'euro" par Benjamin Angel, John Tehuritaua, Maire Liu.

11 Benjamin Angel (membre du cabinet de M. Joaquim Almunia, Commissaire Européen chargé des Affaires Économiques et Monétaires) et Alexandre Czmal (juriste et représentant de l'assemblée des Chambres de Commerce et d'Industrie).

12 Les PTOM ne font pas partie de l'UE, bien qu'ils fassent partie de leur Etat membre de rattachement. Le droit communautaire ne leur est pas applicable, à l'exception du régime d'association basé sur la Partie IV du Traité CE (articles 182 à 188) contrairement aux Départements d'Outre-Mer (DOM) qui font partie de l'UE. 
dans les 3 PTOM du Pacifique. Puis, avec l'aide de la BCE, la Commission négocierait un accord avec la France, dont la délégation comprendrait aussi les représentants des 3 PTOM. L'accord serait vraisemblablement avalisé par le Conseil de l'Union Européenne, avant que la Polynésie française doive adapter son cadre légal conformément à l'accord, et que l'euro y soit introduit. Au total, il faudrait 26 directives, 6 règlements, 9 décision du Conseil, 3 recommandations de la Commission et 5 orientations de la $\mathrm{BCE}$ pour arriver à cet accord final au niveau européen.

Enfin, il faudrait prendre des mesures au niveau de la Polynésie française. D'abord, l'ensemble des textes de loi, des réglementations, des contrats publics et privés, ainsi que des systèmes informatiques liés au franc CFP doit être modifié. Une campagne d'information suivie d'une période de double présentation en francs CFP et euros serait nécessaire pour préparer la population, les entreprises et les administrations au passage à l'euro. En particulier, la crainte majeure exprimée par la population est celle d'une inflation générée par cette transition à une nouvelle monnaie. Nous y reviendrons plus tard dans cet article, mais il est clair qu'il faudrait établir un programme crédible de surveillance des prix pour éviter les dérapages inflationnistes possibles.

Ce sont les raisons pour lesquelles la procédure complète prendrait environ trois ans avant que les pièces et billets soient mis en distribution, avec une période d'échange de la monnaie locale contre des euros comme cela avait été le cas dans la zone euro au début de 2002. Les pièces en euros seront les pièces françaises, mais il semble que la frappe de pièces commémoratives de 2 euros, avec des symboles polynésiens, mais ayant cours légal dans l'ensemble de la zone euro, soit une possibilité.

\section{Contraintes Institutionnelles du Passage à l'Euro}

Bien que la Polynésie française soit considéré comme un PTOM pour l'UE, et donc que le droit communautaire ne lui soit pas applicable, le fait qu'elle adopte l'euro comme monnaie locale la contraindrait à appliquer la législation monétaire et financière européenne, ainsi qu'une partie de la législation fiscale européenne. Mais le rapport de l'Assemblée lors de sa résolution en faveur du passage à l'euro conclut que «les modifications du droit polynésien seraient assez limitées» (2005, page 9).

En effet, la législation monétaire européenne ne concerne que l'introduction de l'euro, les taux de conversion, le système de paiement, les arrondis, les pièces et billets, la lutte contre le faux monnayage, la collecte et la production de statistiques, les pouvoirs de la BCE et les réserves obligatoires. La législation financière européenne est déjà applicable, à travers le code monétaire et financier français qui le transpose en Polynésie française. Il ne resterait qu'à modifier le droit commercial (en particulier, le droit des faillites et le droit des garanties) pour le rendre compatible avec les règles de l'union monétaire européenne.

Seule une partie de la législation fiscale européenne devrait occasionner certaines adaptations significatives de la réglementation locale. En particulier, la fiscalité de l'épargne devrait être modifiée, pour éviter toute discrimination entre résidents polynésiens et investisseurs européens. Par 
exemple, les taux d'imposition sur les intérêts de l'épargne sont actuellement de $25 \%$ en Europe, et de 7\% ou moins en Polynésie française. ${ }^{13}$ L'Etat devrait négocier avec chacune des collectivités du Pacifique pour faire converger ces taux. De plus, l'imposition à la source de $10 \%$ sur les revenus des non-résidents devrait être modifiée, puisque les revenus des résidents ne sont pas taxés directement. Enfin, un code de bonne conduite en matière de fiscalité des entreprises ainsi qu'un système d'échanges de renseignements en matière fiscale devraient être mis en place.

Par contre, la Polynésie française garderait la même neutralité fiscale dont elle bénéficie aujourd'hui. En particulier, elle ne sera pas soumise aux critères de Maastricht, qui définit des contraintes économiques et financières en vue de joindre et de rester dans l'Union Monétaire Européenne (UME), puisque c'est la France qui a cette responsabilité. Rappelons que ces critères concernent le déficit public (limité à $3 \%$ du Produit Intérieur Brut ou PIB), la dette publique (limité à $60 \% \mathrm{du} \mathrm{PIB})$, l'interdiction du financement monétaire des déficits publics, et le taux d'inflation (dont la limite est calculée par rapport au taux d'inflation moyen de la zone euro). De même, il n'y a pas de raisons légales pour lesquelles le passage à l'euro devrait affecter pas les transferts financiers de l'État, qui représentent environ un quart du PIB de la Polynésie française. De plus, la résolution du 19 janvier 2006 conclut par ces remarques importantes:

L'Assemblée précise cependant que l'introduction de l'euro:

- Ne devra absolument pas entraîner une redéfinition de l'actuelle parité franc CFP / euro.

- Ne devra pas porter atteinte aux compétences que reconnaît la loi statutaire du 27 février 2004 à la Polynésie française.

- Implique que l'Etat aide la Polynésie française à supporter le coût de ce changement de régime monétaire.

Il est intéressant de souligner que ces trois conditions ne sont que des souhaits de la part de l'Assemblée. En particulier, la France reste seule habilitée à déterminer la parité du franc CFP, comme le rappelle le protocole spécial $n^{\circ} 13$ du Traité de Maastricht, et elle pourrait en théorie dévaluer le franc CFP à tout moment, par exemple juste avant le passage à l'euro. Cependant, la quasi-totalité des experts et politiciens en Polynésie française et en métropole semblent d'accord sur l'effet néfaste qu'aurait une telle dévaluation sur l'économie locale aujourd'hui. Nous reviendrons sur ce point en détails dans la partie suivante. De même, il paraît fort improbable que la loi statutaire de 2004 ne soit pas respectée. Quant au coût du passage à l'euro, rien n'a été encore négocié avec l'Etat jusqu'à maintenant, puisque les deux PTOM du Pacifique n'ont pas encore officiellement émis le souhait d'introduire l'euro, même si une certaine volonté politique semble présente.

13 Trois sources mentionnent des taux d'imposition différents sur les intérêts de l'épargne: 7\%, 6\% et 4\%. 
En tout cas, la valeur de la nouvelle monnaie locale continuerait à être assurée, mais cette fois par la BCE. La Banque de France gardera le privilège d'émission de la monnaie locale (l'euro au lieu du franc CFP), mais le sort de l'Institut d'Émission d'Outre-Mer (IEOM) semble par contre plus incertain. Il pourrait soit disparaître, soit être converti en Institut d'Emission des Départements d'Outre-Mer (IEDOM) comme pour Mayotte ou Saint Pierre et Miquelon, soit être considéré comme une succursale de la Banque de France dans le Pacifique. Par contre, il est assuré que le mécanisme de réescompte employé par l'IEOM pour aider au refinancement des établissements de crédit, ce qui ne concerne que $2 \%$ des crédits consentis localement, devrait disparaître. Cette suppression d'un financement privilégié pour les entreprises des secteurs considérés comme prioritaires serait probablement compensée par l'amélioration des conditions de crédit dus à la nouvelle rémunération des réserves obligatoires (i.e., alignement sur le régime des réserves obligatoires imposé par le Système Européen des Banques Centrales) et à la nouvelle concurrence directe avec les autres banques européennes. En effet, le taux de base bancaire en Polynésie française, qui a été supérieur d'environ $1 \%$ ou $2 \%$ à celui observé en métropole, devrait baisser en convergeant vers ce dernier.

Ainsi, les modalités et contraintes institutionnelles ne semblent pas être un obstacle majeur pour l'introduction de l'euro en Polynésie française. Les problématiques du passage à l'euro se situent plus dans un contexte économique et demandent une analyse microéconomique et macroéconomique pour bien comprendre les enjeux de cette importante décision.

\section{LES ENJEUX ÉCONOMIQUES DU PASSAGE À L'EURO}

\section{A Historique des Régimes de Change en Polynésie Française}

Le taux de change entre le franc CFP et la monnaie en métropole est resté fixe, sans aucune dévaluation depuis plus de 57 ans, ce qui constitue probablement un record. Pendant cette longue période, le système monétaire international a vécu de profonds changements. De 1949 à 1971, le système monétaire de Bretton-Woods avait établi des parités fixes autour du dollar US, mais avec des possibilités d'ajustements (ie, dévaluations par rapport à la monnaie américaine). En particulier, le franc français, et donc le franc CFP, ont perdu environ 40\% de leur valeur à cause de dévaluations majeures de ce premier par rapport au dollar US, telles qu'en 1958 et 1969. Le 15 août 1971, le président Nixon déclarait la fin de la convertibilité du dollar US en or, et marquait ainsi la fin de la période de Bretton-Woods. Malgré quelques efforts pour le sauver (y compris deux dévaluations du dollar) et le rôle de monnaie internationale que tenait (et tient toujours) le dollar, ce système ne survivra pas aux déséquilibres macroéconomiques de l'époque, c'est-à-dire un large déficit commercial des États-Unis d'Amérique. Cela démontre qu'aucune monnaie, si puissante qu'elle soit, ne peut résister indéfiniment à des déséquilibres excessifs dans la balance des paiements. Il est important de souligner que cette leçon d'économie internationale s'applique toujours aujourd'hui au dollar, même si les États-Unis laissent leur taux de change flotter depuis 1973 sans intervenir souvent dans les marchés de change pour le stabiliser (sauf en 1985 et 1987). En effet, le déficit du compte courant Américain a atteint des sommets jamais atteints (même en pourcentage du PIB), et de nombreux économistes s'attendent à une chute sévère de la valeur du dollar, qui s'est déjà 
entamée depuis l'an 2002. Nous reviendrons plus tard sur les énormes déficits commerciaux de la Polynésie française depuis 1960 et les raisons pour lesquelles sa monnaie a échappé à une dévaluation depuis si longtemps.

\section{B Le Choix d'un Régime de Change Optimal et Son Application à la Polynésie Française}

Il est utile de rappeler qu'il existe différents types de régimes de change parmi lesquels la Polynésie française a tout intérêt à trouver le régime optimal pour son économie très particulière:

- Les taux de change flottants (ou flexibles) sont déterminés uniquement par l'offre et la demande dans les marchés de change. Les économistes libéraux considèrent que ces marchés ajustent rationnellement et d'une façon optimale les taux de change à leur équilibre, alors que les économistes dits Keynésiens critiquent la volatilité excessive des taux de change dus à une spéculation déstabilisante. En tout cas, tous sont d'accord sur le fait que ce régime de change flexible permet à la banque centrale d'avoir une politique monétaire indépendante et puissante. C'est en particulier le cas des Etats-Unis, puisqu'ils ont adopté une politique de taux de change flexibles en intervenant dans les marchés de change que très rarement depuis 1973.

- Les régimes de taux de change intermédiaires sont des régimes hybrides entre régimes de change flottants et régimes de change fixes. Ils bénéficient d'une partie des avantages des deux systèmes, mais souffrent aussi d'une partie de leurs faiblesses. Bien que préconisés par certains économistes pour des pays émergents, ces régimes sont en général transitoires. La politique monétaire y est limitée, mais la politique de change est active sans être dogmatique. On peut citer comme exemple les cibles de taux de change, tel que les «baskets, bands, crawls» (BBC).

- Les taux de change fixes (par rapport à une ou plusieurs monnaies étrangères, telles que le dollar US et maintenant l'euro) sont contrôlés par le gouvernement local, qui doit acheter et vendre des réserves internationales pour équilibrer l'offre et la demande au taux de change désiré. Les économistes Keynésiens voient dans ce régime une solution pour stabiliser les taux de change dans le court terme, alors que les économistes libéraux soulignent que les nombreuses crises de régime de change fixe (lorsque les dévaluations sont provoquées par des attaques spéculatives) sont plus dévastatrices que les fluctuations de taux de change flexibles. En tout cas, tous sont d'accord sur le fait que ce régime de change fixe permet d'ancrer les prix (et taux d'intérêt) locaux aux prix (et taux d'intérêt) étrangers, mais supprime l'indépendance de la politique monétaire en l'absence de contrôle de capitaux. Ce régime de taux de change fixes, mais ajustables (lorsque les dévaluations sont désirées) a été choisi par la plupart des pays pendant la période de Bretton-Woods (1949-1971) et par un nombre décroissant de pays depuis 1973. C'est le régime de change qui est en vigueur en Polynésie française depuis 1949, sans avoir souffert d'une seule dévaluation. C'est aussi le cas de la Chine, qui a fixé la valeur de sa monnaie par rapport au dollar US jusqu'en juillet 
2005, et par rapport à un panier de monnaies depuis cette date. Cependant, contrairement à la quasi-totalité des autres pays, la Chine continue à exercer un strict contrôle sur les flux de capitaux entrants et sortants, et peut donc bénéficier simultanément d'une politique monétaire indépendante et d'un régime de change fixe.

- Au-delà des régimes de taux de change fixes et ajustables, il existe des régimes de taux de change "rigides", ainsi nommés car ils sont configurés pour éviter à tout prix une dévaluation. Premièrement, les caisses d'émission sont des quasi-banques centrales dont le but unique est de maintenir une certaine parité entre la monnaie locale et une monnaie étrangère (tels qu'à Hong Kong par rapport au dollar US, ou en Bulgarie par rapport à l'euro $\left.^{14}\right)$. L'Argentine fournit un excellent exemple de ses avantages et inconvénients, puisqu'elle a réussi très rapidement à éliminer son hyperinflation en instaurant une caisse d'émission en 1991, mais elle a dû abandonner ce régime de change en 2001 et laisser sa monnaie ainsi que son économie s'effondrer dans le court terme à cause de l'impossibilité d'utiliser une politique monétaire dans ce régime de change rigide. Deuxièmement, les unions monétaires consistent à adopter une monnaie étrangère (tels qu'au Panama avec le dollar US), ou à partager une monnaie commune entre différents pays (tels que dans l'UME avec l'euro). Ce régime extrême est à priori irrévocable, à moins de recréer une nouvelle monnaie locale dans son pays. Ses avantages majeurs sont l'élimination de toute crainte de fluctuations de change dans la zone monétaire et la promotion du commerce intra-zone, alors que ses inconvénients principaux sont l'abandon définitif d'une politique monétaire individuelle et la soumission à d'autres contraintes économiques (tels que les critères de Maastricht pour l'UME). Enfin, les économies "dollarisées" (surtout en Amérique Latine) ou "euroisées" (surtout les micro-États d'Europe) ${ }^{15}$ sont caractérisées par une forte circulation (dans les marchés) ou utilisation (dans les contrats) de monnaie étrangère en parallèle avec la monnaie locale. Ce régime hybride semble réduire les risques de crises de change, mais aussi augmenter les risques de crises bancaires. ${ }^{16}$

Puisque le taux de change du franc CFP est aujourd'hui fixe par rapport à l'euro, le choix d'un régime de change se résume à: (i) un statu quo; (ii) un rattachement à l'UME (c'est-à-dire le remplacement du franc CFP par l'euro); (iii) un régime de taux de change purement flexible; (iv) un

14 Anne-Marie Gulde, Juha Kahkönen, Peter Keller, (2000) "Pros and Cons of Currency Board Arrangements in the Lead-up to EU Accession and Participation in the Euro Zone". International Monetary Fund Policy Discussion Paper 00/01.

15 Adalbert Winkler, Francesco Mazzaferro, Carolin Nerlich, Christian Thimann (2004), "Official Dollarisation/Euroisation: Motives, Features and Policy Implications of Current Cases", European Central Bank Occasional Paper Series, 2004-11.

16 Anne-Marie Gulde, David Hoelscher, Alain Ize, David Marston, Gianni De Nicoló, "Financial Stability in Dollarized Economies" (2004) International Monetary Fund Occasional Paper 230. 
régime de taux de change intermédiaire, avec intervention sur les marchés de change; (v) un régime de taux fixe avec une autre monnaie telle que le dollar US ou le yen japonais (c'est-à-dire les monnaies des deux autres principaux partenaires commerciaux); (vi) une union monétaire avec l'une de ces deux monnaies (c'est-à-dire le remplacement du franc CFP par le dollar ou le yen).

Sans considérer les arguments politiques (qui sont actuellement en faveur du rattachement à l'UME) et en se basant purement sur des raisonnements économiques, on d'abord observer qu'en 2005, la part de la France (35\%) dans les pays fournisseurs des importations polynésiennes (dont la moitié correspond à des produits mécaniques et électriques) est clairement supérieure à celle des Etats-Unis (10\%). La part importante du Japon (28\%) et de Hong Kong (28\%) dans les pays de destinations des exportations polynésiennes s'explique par la prédominance des perles dans ces exportations (75\%). De plus, les exportations ne représentent qu'environ $11 \%$ des importations pour les biens civils, mais 50\% des importations pour les biens et services privés en 2004. Enfin, la totalité des transferts publics, qui est équivalent à environ un quart du PIB polynésien et qui a toujours financé une très grande partie des déficits de biens et de services privés, provient de métropole. Par conséquent, la France représente $63 \%$ de toutes les transactions du compte courant (somme des flux d'exportations et d'importations de biens et de services, ainsi que de revenus et de transferts) et $97 \%$ de toutes les transactions du compte financier de la Polynésie française. La logique économique demande donc d'éliminer les deux dernières options, et de ne garder que les quatre premières options, dont deux possèdent l'euro comme référence pour le choix d'un régime de change optimal pour la Polynésie française.

En vue de choisir ce régime de change optimal, une théorie économique a été développée sous le nom de "critères de zones monétaires optimales" (par Robert Mundell, ${ }^{17}$ ce qui lui a valu, avec d'autres travaux de recherche, un prix Nobel en économie), puis récemment réformée. ${ }^{18}$ Les critères traditionnels de zones monétaires optimales sont des conditions ex ante (qui précèdent l'adoption d'une union monétaire), alors que les nouveaux critères de zones monétaires optimales sont des avantages ex post (qui sont des conséquences possibles d'une union monétaire). Ainsi, ces deux théories ne sont pas nécessairement contradictoires. Cependant, il n'existe pas de consensus sur la supériorité d'une théorie sur l'autre, ${ }^{19}$ et il est important d'examiner leur application respective à la Polynésie française avant d'en tirer des conclusions toutefois nettes et claires.

Les critères traditionnels pour faire partie d'une zone monétaire optimale sont les suivants:

- Flexibilité des prix et des salaires: en Polynésie française, les prix de certains produits, appelés jusqu'ici produits de première nécessité (PPN) sont fixés par le gouvernement local,

17 Robert Mundell "A Theory of Optimum Currency Areas" (1961) 51 American Economic Review 657-665.

18 Jeffrey Frankel, Andrew Rose "The Endogeneity of the Optimum Currency Area Criteria" (1998) 108 Economic Journal 1009-1025.

19 Alberto Alesina, Robert Barro "Currency Unions" (2002) 117 Quarterly Journal of Economics 409-436. 
mais leur nombre et leur poids de l'indice des prix à la consommation est relativement faible. Cependant, les salaires des fonctionnaires d'Etat et par extension des fonctionnaires locaux sont "indexés" à un niveau très élevés (d'environ $80 \%$ supérieur à ceux de la métropole). L'importance du secteur public dans l'économie polynésienne pousse donc les salaires moyens polynésiens et donc les prix (par un effet de demande accrue) vers le haut. De plus, la concurrence avec les produits importés n'est pas très forte, puisque la production locale est différenciée. On peut en conclure que la flexibilité des prix et des salaires vers le bas est relativement faible, et qu'un choc asymétrique avec la zone euro ne pourrait être compensé par une politique monétaire indépendante.

- Mobilité des facteurs de production (travail, capital): la Polynésie française, à cause de sa situation géographique très isolée, mais aussi de sa qualité de vie élevée, n'est pas caractérisée par des flux migratoires importants, contrairement aux autres îles du Pacifique Sud qui sont nettement plus pauvres. Les polynésiens, même si leur passé culturel révèle un instinct de grand voyageur, n'ont pas vraiment eu de raisons qui les poussaient à s'expatrier, depuis le bond en avant économique des années soixante. L'ouverture du Centre d'Expérimentation du Pacifique (CEP) a par contre provoqué une immigration temporaire de militaires et d'administrateurs métropolitains vers la Polynésie française pendant cette décennie, mais comparativement peu d'émigrants travaillant dans le secteur privé. De même, les mouvements nets de capitaux privés vis-à-vis de l'extérieur sont négligeables quand on les compare aux transferts financiers de l'État, qui représentent aujourd'hui environ un quart du revenu total de la Polynésie française. On peut en déduire que la mobilité des facteurs de production vers ou en provenance de l'extérieur est faible dans le secteur privé, mais forte dans le secteur public, ce qui donne à la Polynésie française un amortisseur de choc asymétrique avec la zone euro.

- Diversification de la production: la petite taille économique de la Polynésie française, avec environ 250.000 habitants, ne lui permet pas de diversifier sa production. En excluant le secteur public du Produit Intérieur Brut (PIB) total, on obtient un PIB marchand (PIBm) de 324,4 milliards de francs CFP en 2002 (dernier chiffre officiel disponible à ce jour), soit à peine $0,3 \%$ du PIB marchand de la métropole. Le secteur principal de l'économie polynésienne est le secteur tertiaire (services), qui représente $73,7 \%$ du PIBm, alors que les secteurs primaire (agriculture, pêche, aquaculture, perliculture) et secondaire (industrie, artisanat) ne représentent respectivement que $4,4 \%$ et $14,9 \%$ du PIBm. La première source de recettes d'exportations vient du tourisme avec 12,4\% du PIBm (avec environ un tiers de touristes en provenance de la zone euro et un tiers en provenance des Etats-Unis), suivi de la perliculture avec 4,6\% du PIBm et des autres exportations civiles avec 1,9\% du PIBm. On voit donc que la production de biens et de services n'est pas diversifiée, en particulier pour ses exportations, ce qui est un défi commun aux petites économies insulaires. 
- Intégration fiscale: bien que le gouvernement de Polynésie française dispose légalement d'une autonomie fiscale importante, il est évident que les transferts financiers de l'État, qui sont estimées à plus de la moitié des dépenses publiques locales, représentent une somme qu'il n'a pas besoin de financer à l'aide d'impôts, de taxes et d'emprunts. D'un point de vue purement économique, il existe donc une forte intégration fiscale entre Polynésie française et la métropole.

- Ouverture des marchés et intégration au commerce international: depuis la fin du choc CEP dans les années soixante, le poids des exportations polynésiennes de biens et services privés dans le PIB a augmenté de $12 \%$ dans les années soixante à $21 \%$ depuis l'an 2000 alors que le poids des importations polynésiennes de biens et services privés dans le PIB a varié de $66 \%$ à $43 \%$ dans ces mêmes périodes. Il faut savoir également que la pondération des produits et services importés dans l'indice des prix à la consommation est d'environ 47\%. En particulier, la grande majorité des biens manufacturés doivent être importés. Cela démontre le haut degré d'ouverture et de dépendance aux marchés internationaux, et plus particulièrement visà-vis de la métropole, qui fournit plus d'un tiers des marchandises importés.

- Intégration des marchés financiers: bien que l'IEOM assure la convertibilité illimitée du franc CFP en euro, le franc CFP n'est pas accepté à l'étranger, ni dans les banques métropolitaines. Même la Banque de France n'échange plus les francs CFP qu'à son siège social à Paris. Plus grave encore, le change entre le franc CFP et l'euro n'est plus gratuit en Polynésie française, alors qu'il l'était entre le franc CFP et le franc français. Cela nuit à la liquidité des flux financiers avec l'extérieur (d'où une faible intégration financière internationale), ainsi qu'à la concurrence avec le secteur bancaire de métropole, et au tourisme. Cependant, adopter l'euro pourrait rapidement augmenter cette intégration financière et concurrence avec la métropole.

- Corrélation des cycles économiques: la corrélation des taux de croissance (du PIB réel) de la Polynésie française est de $35 \%$ avec la métropole. Une analyse plus fine montre que l'absence relative d'asymétrie des chocs économiques avec la métropole est un avantage pour adopter l'euro.

Les nouveaux critères endogènes d'une zone monétaire optimale ne sont pas des conditions à remplir avant de joindre une union monétaire, mais plutôt des estimations empiriques des bénéfices à tirer de cette union monétaire. Les deux principaux critères endogènes sont les suivants:

- Inefficacité des dévaluations: depuis 1960, la Polynésie française a continuellement eu un déficit de la balance des biens et des services privés (c'est-à-dire, la balance courante en excluant tous les transferts nets de l'État). Sous l'effet expansionniste du choc CEP, ce déficit a explosé pour dépasser les $80 \%$ du PIB en 1966, puis il a progressivement, mais lentement diminué jusqu'à brièvement descendre en dessous de $10 \%$ du PIB en 1999 , 
d'après des extrapolations basées sur des concepts de comptabilité nationale ${ }^{20}$. Il est ensuite remonté jusqu'à environ $20 \%$ ces dernières années. Sans entrer dans des détails techniques, cela signifie qu'une dévaluation aurait un effet inflationniste sur les importations plus grand que l'effet de compétitivité sur les exportations. Pour cette raison, supprimer le risque d'une telle dévaluation potentiellement néfaste en faisant partie de l'UME (puisqu'un tiers des importations proviennent de métropole) serait bénéfique.

- Crédibilité de la banque centrale: le risque de dévaluation précédemment discuté plane toujours au dessus de la Polynésie française, puisqu'il suffit d'un décret du gouvernement français pour changer la parité du franc CFP par rapport à l'euro. Remplacer le franc CFP par l'euro irrévocablement reviendrait donc à éliminer cette possibilité et à bénéficier de la crédibilité de la BCE.

L'évaluation des critères traditionnels d'une zone monétaire optimale montre que certaines de ces conditions (flexibilité des prix et des salaires, mobilité des facteurs privés de production, diversification de la production, intégration des marchés financiers) ne sont pas remplies en ce moment, mais que la plupart des autres critères traditionnels (intégration fiscale, ouverture des marchés et intégration au commerce international, corrélation des cycles économiques), ainsi que les critères endogènes (inefficacité des dévaluations, crédibilité de la banque centrale), sont satisfaits. Parmi tous ces critères, l'importante intégration économique et financière avec la métropole, dus aux énormes transferts publics, semble être un facteur déterminant pour conclure qu'une union monétaire avec l'euro est optimale. En effet, le déficit de la balance des biens et services privés est financé en grande partie par les transferts de l'Etat français: c'était déjà l'une des raisons principales pour privilégier l'euro plutôt que le dollar US ou le yen. C'est également la cause majeure du rejet d'un régime de taux de change flexible ou intermédiaire. Sans l'intervention inconditionnelle de l'IEOM, qui dispose actuellement de la possibilité d'un découvert illimité auprès du Trésor Public pour assurer la convertibilité du franc CFP à la même parité depuis 1949, il est fort probable que le franc CFP se déprécierait rapidement, à cause du déficit chronique de la balance des biens et services privés et de la crainte de manque de financement de ce déficit par les transferts de l'État. Cette quasi-dévaluation provoquerait d'importantes variations asymétriques entre les crédits (exportations et transferts de l'État) et les débits (importations) dans la balance des paiements, et créerait ainsi des déséquilibres financiers qui pourraient déstabiliser l'économie polynésienne. Un argument similaire pourrait être invoqué contre le choix d'un régime de taux de change intermédiaire, tel qu'une cible de taux de change réel, puisque l'expérience dans d'autres pays suggère que les interventions dans les

20 Depuis 1998, l'IEOM fournit des estimations fiables sur les diverses composantes de la balance des paiements: d'après ces estimations, le même déficit de la balance des biens et des services privés en 1999 s'élevait à $15,3 \%$ du PIB. En 1995, il était remonté à 23,4\% du PIB. La différence entre ces ratios et ceux présentés ci-dessus tiennent à ce que les comptes économiques de Polynésie française ne tenaient pas compte jusqu'à présent des importations de services, tels que les voyages à l'étranger par les résidents polynésiens. 
marchés des changes réussissent rarement à maintenir un taux de change dans une cible déterminée dans le long terme, et donc que ce régime ne résiste en général pas longtemps aux attaques spéculatives ou aux fuites de capitaux quand il existe un déficit externe (privé) important comme en Polynésie française. Il reste donc à trancher entre les deux premières options: (i) le maintien du régime de change fixe actuel; (ii) l'introduction de l'euro en remplacement du franc CFP. L'analyse suivante des effets micro-économiques et macro-économiques du passage à l'euro nous donnera la réponse à cette question.

\section{Les Effets Microéconomiques du Passage à l'Euro}

La microéconomie traite du comportement individuel des agents économiques, de leurs interactions, et de la nature des marchés dans lesquels ils opèrent. Cette science aide les consommateurs, les producteurs, les investisseurs, et les décisionnaires politiques à faire des choix optimaux. Elle s'applique dans une certaine mesure, en particulier dans le court terme, à une décision telle que celle du régime de change en Polynésie française. En effet, l'introduction de l'euro en remplacement du franc CFP aurait des effets immédiats sur les paramètres de l'économie polynésienne et sur les décisions de ses acteurs. Nous allons donc examiner les trois principaux effets du passage à l'euro, en les contrastant avec le statu quo, c'est-à-dire le maintien du régime de change fixe actuel entre le franc CFP et l'euro.

La première conséquence de la substitution du franc CFP par l'euro serait l'élimination du risque de dévaluation. En raisonnant a contrario, on peut d'abord montrer qu'une dévaluation serait néfaste sur l'économie polynésienne, comme il a été brièvement décrit lors de la discussion des critères endogènes d'une zone monétaire optimale, et donc révéler un premier avantage en faveur du passage à l'euro. Un examen plus approfondi des effets économiques d'une dévaluation du franc CFP appartient à l'analyse macroéconomique, et sera donc présenté dans la section suivante. Ensuite, l'élimination du risque de dévaluation ferait diminuer les primes de risque de change et donc les taux d'intérêts. En effet, les taux de base bancaire en Polynésie française ont été d'environ $1 \%$ plus élevés qu'en métropole au cours des dix dernières années, en partie à cause de ce risque de change, même s'il n'y a eu aucune dévaluation depuis presque soixante ans. Il existe bien des prêts à taux bonifiés, consentis à certaines entreprises prioritaires via le mécanisme de réescompte, ou aux investisseurs immobiliers via une subvention du gouvernement locale, mais seuls les bonifications dus au mécanisme de réescompte, qui ne concernent que $2 \%$ des crédit totaux, seraient supprimées. De plus, l'adoption de l'euro, qui est aujourd'hui la monnaie commune dans les douze pays de l'UME (regroupant plus de 300 millions de personnes) ainsi que dans de nombreux autres états, procurerait un accès direct aux marchés de capitaux internationaux. Cette ouverture aux marchés financiers internationaux augmenterait la concurrence bancaire et ferait également baisser les différentiels de taux d'intérêts par rapport à ceux pratiqués dans la zone euro. Par conséquent, la baisse du risque et du coût de l'emprunt provoquerait une hausse des investissements locaux et des influx de capitaux extérieurs en Polynésie française, ce qui stimulerait son économie. 
La seconde répercussion de l'adoption de l'euro serait la facilitation des échanges commerciaux avec le reste du monde. Le remplacement d'une monnaie essentiellement inconvertible à l'étranger (c'est-à-dire le franc CFP) par une monnaie non seulement convertible, mais aussi à forte crédibilité internationale (c'est-à-dire l'euro) favoriserait les exportations, et en particulier le tourisme (exportation de services), qui est la source principale de revenus extérieurs privés. De plus, les coûts de transactions (en particulier, les commissions de change) seraient sensiblement réduits et la comptabilité serait simplifiée pour le commerce extérieur, ce qui l'inciterait à se développer encore plus rapidement. Il existerait bien des coûts de transition à l'euro pour les institutions publiques locales et les entreprises privées, mais nous avons vu auparavant que l'Assemblée souhaitait dans son rapport ${ }^{21}$ que l'introduction de l'euro «implique que l'Etat aide la Polynésie française à supporter le coût de ce changement de régime monétaire». Au vu de l'expérience dans d'autres DOM-TOM, il semble que ces coûts de transition ne soient pas très élevés, en comparaison des bénéfices de long terme.

En tout cas, il faut savoir qu'une politique de croissance basée sur les exportations (dont le tourisme reste la pierre angulaire en Polynésie française), est souvent la seule politique vraisemblable à mener pour une petite économie insulaire ${ }^{22,23}$, à moins d'avoir des ressources exceptionnelles, comme le nickel en Nouvelle Calédonie ${ }^{24}$. De plus, la zone euro, bien qu'elle ne pèse que $15 \%$ du PIB mondiale (contre $20 \%$ pour les États-Unis), est responsable pour $30 \%$ des exportations de biens et de services dans le monde (contre $10 \%$ pour les États-Unis) ${ }^{25}$. Adopter l'euro permettrait de faciliter les échanges commerciaux non seulement avec la zone euro, mais avec le reste du monde, puisque la monnaie européenne est devenue la seconde monnaie d'échanges commerciaux sur le plan international.

Le troisième effet microéconomique du passage à l'euro serait l'amélioration de la transparence des prix relatifs à l'étranger. En effet, la population polynésienne pourrait beaucoup plus facilement comparer les prix en Polynésie française et en métropole, puisqu'il serait exprimé dans la même

21 Assemblée de la Polynésie Française, Commission des Finances (19 janvier 2006), "Rapport concernant une proposition de résolution relative à l'introduction de l'euro en Polynésie Française", nº 011-2006.

22 Bernard Poirine Les petites économies insulaires: théories et stratégies de développement (L'Harmattan, Paris, 1995).

23 Hong Kong et Hawaii sont des exemples de réussite économique due respectivement à une telle politique d'ouverture commerciale et à une promotion intense du tourisme (le nombre annuel de touristes est d'environ 7 par habitant, alors qu'il ne dépasse pas 1 en Polynésie française). Hong Kong a également adopté en 1984 une caisse d'émission pour sa monnaie (le dollar de Hong Kong) qui est depuis liée au dollar US, alors que Hawaii, en tant que 50ème état américain, utilise évidemment ce même dollar US.

24 Institut d'Emission d'Outre-Mer (2006) "La Nouvelle Calédonie en 2005" Rapport Annuel.

25 Source: International Monetary Fund "World Economic Outlook" (2006). 
monnaie. L'accès à internet, en particulier en haut débit (ADSL), croît rapidement $(+22 \% \text { en 2005) })^{26}$ et permet non seulement de comparer les prix, mais aussi de commander les mêmes produits que ceux disponibles en Polynésie française. Cela ferait donc augmenter la concurrence et induirait une baisse des prix. On pourrait rétorquer que les exportations de produits polynésiens pourraient souffrir d'une perte de compétitivité internationale en cas d'appréciation de l'euro, mais il n'y aurait de ce point de vue aucune différence avec le régime actuel de taux de change fixe vis-à-vis de l'euro (en faisant bien sûr l'hypothèse d'une absence de dévaluation).

Il faut enfin évoquer une crainte majeure parmi la population en Polynésie française, comme l'ont montré les débats au CESC et à l'Assemblée: la possibilité de dérapage des prix lors de la transition à l'euro. Un exemple simple suffit à décrire cette crainte. Si le prix d'une voiture est 1.000.000 francs CFP, alors son prix est exactement 8380 euros, car le taux de change officiel est 1000 francs CFP $=8,38$ euros. Si le prix d'un coco glacé (un produit qui est souvent proposé par un vendeur ambulant) est 100 francs CFP, alors son prix devrait être exactement 0,838 euros, mais le prix devra être officiellement arrondi à 0,84 euros, d'où une inflation artificielle de $(0,84$ $0,838) / 0,838=0,2 \%$. (Par coïncidence, ce pourcentage est aussi l'augmentation d'inflation due au passage à l'Euro en métropole !). Cependant, un vendeur ambulant aura peut-être tendance à arrondir le prix à 1 euro, d'où une inflation artificielle de $(1,00-0838) / 0,838=19,3 \%$. On comprend donc que la population perçoive des risques d'inflation supplémentaire et soit méfiante envers le remplacement du franc CFP par l'euro. De plus, les média ont montré que la plupart des métropolitains (et des européens) étaient convaincus d'une montée des prix à cause du passage à l'euro, ce qui a ajouté à la psychose. Or, les analyses détaillées de la $\mathrm{BCE}^{27}$ et des diverses banques centrales de la zone euro ont démontré l'absence d'un effet inflationniste significatif lors du passage à l'euro (sauf dans quelques secteurs d'activités, tels que la restauration et le cinéma), contrairement aux idées perçues. En ce qui concerne la Polynésie française, les dépenses alimentaires représentaient $18.9 \%$ des dépenses totales ${ }^{28}$ en 2000 , et les dépenses de produits proposés par des vendeurs ambulants ne représentent qu'une faible portion des dépenses alimentaires, d'où une inflation artificielle faible en réalité. Une obligation de double affichage des prix en francs CFP et en euros (dans les magasins), ainsi qu'une politique de lutte contre des arrondis abusifs pourraient empêcher un dérapage des prix lors de la transition à l'euro. Enfin, les billets et pièces en franc CFP sont parés de beaux symboles polynésiens et calédoniens, dont la population est naturellement fière. Il faudrait donc une campagne d'information judicieuse pour rassurer les polynésiens.

26 Institut d'Emission d'Outre-Mer (2006) "La Polynésie Française en 2005" Rapport Annuel.

27 Michael Ehrmann, "Rational inattention, inflation developments and perceptions after the Euro cash changeover" (2006) European Central Bank Working Paper 588.

28 Institut de la Statistique de Polynésie Française (2002), "Etude Budget des Familles". 


\section{L'équilibre Macro-économique et le Passage à l'euro}

La macroéconomie traite des relations entre agrégats économiques intérieurs (croissance, chômage, inflation, ...), mais aussi extérieurs (balance commerciale ou courante, taux de change, ...). Elle analyse également les effets des politiques économiques (politique fiscale, politique monétaire, politique de change, politique commerciale). Ces effets varient selon le régime de change choisi, ${ }^{29}$ et il faut donc examiner en détail les contraintes et conséquences macroéconomiques de l'introduction de l'euro en remplacement du franc CFP.

Il faut d'abord savoir qu'en l'absence de contrôles de capitaux, qui ont été aboli en 1990 dans la France entière (en préparation du traité de Maastricht), il est impossible d'utiliser dans le long terme une politique monétaire indépendante sous un régime de change fixe. Sous le régime passé entre le franc CFP et le franc français, la politique monétaire de Polynésie française, en particulier les taux d'intérêts directeurs, était donc dictée par la Banque de France, et assurée par l'IEOM. Sous le régime actuel entre le franc CFP et l'euro, cette politique monétaire est maintenant dictée par la BCE, même si la Banque de France garde la responsabilité de mener à bien cette politique dans la France entière, y compris la Polynésie française via l'IEOM. A l'exception des changements institutionnels décrits précédemment (en particulier, en ce qui concerne le futur statut de l'IEOM et la suppression du mécanisme de réescompte), la seule différence du point de vue macroéconomique entre le régime actuel de change fixe par rapport à l'euro et le remplacement du franc CFP par l'euro réside dans l'élimination de toute possibilité de dévaluation dans le futur. Pour comprendre l'importance cruciale de cette différence, il faut analyser la balance des paiements de Polynésie française, ainsi que le rôle des taux de change.

La balance des paiements de la Polynésie française, dont les composantes sont estimées depuis 1998 par l'IEOM ${ }^{30}$, décrit l'ensemble des mouvements financiers et non financiers (dont les flux publics en italiques) entre les résidents polynésiens et les non-résidents. Elle consiste en un compte courant (c'est-à-dire, les exportations nettes, pour être bref), un compte financier (c'est-à-dire, les flux nets de capitaux) et un compte de réserves internationales, dont leur somme est toujours égale à zéro, soit la différence entre la demande et l'offre de francs CFP. Si les taux de change du franc CFP étaient flexibles, alors ils équilibreraient la balance des paiements automatiquement, puisque la

29 Robert Mundell (1962) "On the appropriate use of monetary and fiscal policy for internal and external balance", IMF Staff Papers, vol 9, 70-79. Un an après avoir publié son fameux article sur les critères de zones monétaires optimales, ce lauréat du prix Nobel a crée une nouvelle branche de la macroéconomie, celle des économies ouvertes, avec ce nouvel article traitant de l'efficacité respective des politiques monétaires et fiscales sous différents régimes de change. Aujourd'hui, on connaît aussi ces préceptes sous le nom de «trinité impossible», ce qui signifie qu'un pays ne peut que choisir deux instruments de politique économique sur les trois suivants: (i) une politique monétaire indépendante; (ii) un régime de taux de change fixe; (iii) une mobilité parfaite des capitaux.

30 Institut d'Emission d'Outre-Mer (2006) "La Balance des Paiements de la Polynésie Française en 2005", Rapport Annuel. 
demande de monnaie domestique (somme des crédits) serait égale à l'offre de monnaie domestique (somme des débits) sur les marchés de change. Dans ce cas, le compte de réserves internationales serait quasiment nul, puisqu'il n'y aurait pas besoin d'intervention de part de la banque centrale sur ces marchés des change. Mais le taux de change du franc CFP est fixe par rapport à l'euro, et l'IEOM ou la Banque de France devrait en théorie intervenir sur le marché de change pour équilibrer l'offre et la demande de francs CFP en vue de maintenir la parité fixe: vendre des réserves internationales lorsque la demande de francs CFP est inférieur à son offre (compte de réserves internationales négatif) ou vice versa. Si un large déficit de la balance courante surgissait sans que la balance financière rééquilibre leur somme, le compte de réserves internationales s'effondrerait, la banque centrale devrait abandonner la parité fixe, et la valeur de la monnaie locale serait dévaluée, comme cela a été le cas lors de nombreuses crises de change ${ }^{31}$. Mais la Polynésie française bénéficie d'un statut monétaire spécial, puisqu'en fait, l'Etat ajuste tous les dix jours les déséquilibres de la balance des paiements de la Polynésie française, et l'IEOM n'a même pas besoin de maintenir des réserves internationales comme le ferait tout autre pays. Plus précisément, le rapport annuel de l'IEOM précise que:

[L'IEOM] assure dans ce cadre l'exécution des transferts de fonds publics et privés entre sa zone d'émission et le reste de la zone euro. Ces mouvements transitent par le compte d'opérations ouvert au nom de l'IEOM dans les livres du Trésor public, le compte d'opérations garantissant de manière illimitée la convertibilité du F CFP en euro. (page 205).

Cette convertibilité illimitée du franc CFP revient à éliminer la contrainte extérieure de la Polynésie française. Autrement dit, l'IEOM peut maintenir la parité du franc CFP vis-à-vis de l'euro (ou du franc français avant 2002), car il peut recourir à un découvert illimité auprès de l'Etat pour rééquilibrer la demande et l'offre de francs CFP, par exemple en cas de déficit de la balance du compte courant qui ne serait pas compensé par un surplus de la balance du compte financier. Il convient de remarquer que cette balance du compte financier est du même ordre que les erreurs et omissions usuelles, et que l'intérêt de l'analyse repose sur les composantes de la balance courante. Cette dernière consiste en la balance commerciale (différence entre exportations et importations de biens), la balance des services (différence entre exportations et importations de services, tels que le tourisme et les transports internationaux, mais aussi les crédits dus aux dépenses nettes des armées françaises en Polynésie française), la balance des revenus (revenus nets des investissements privés, mais aussi les crédits dus aux rémunérations nettes des fonctionnaires de l'Etat mutés en Polynésie française), la balance des transferts courants (transferts privés nets, mais aussi les crédits dus aux transferts des administrations publiques, dont les retraites de fonctionnaires de l'Etat en Polynésie française).

31 André Cartapanis "Le déclenchement des crises de change: qu'avons-nous appris depuis dix ans?" (2004) 97 Economie Internationale 5-48. 
Comme on peut le voir dans le tableau ci-dessous, le compte courant en 2005 est pratiquement équilibré, mais cet agrégat cache un énorme déficit commercial de 132,5 milliards de francs CFP, qui représente environ 26\% du PIB polynésien (estimé à environ 525 milliards de francs CFP en 2005). Pour donner une idée de l'ampleur de ce déséquilibre, on peut le comparer au déficit commercial des Etats-Unis, qui a atteint son record en 2005 avec 6,4\% du PIB américain, et dont la plupart des économistes s'inquiètent, de crainte de voir le dollar US s'effondrer. En effet, la plupart des crises de change observées dans le monde (à l'exception des crises dites de seconde ou troisième génération, comme la crise asiatique ${ }^{32}$ ) sont le résultat de déficits commerciaux qui avoisinent ou dépassent les $8 \%$ du PIB, car ils engendrent des déficits courants du même ordre, ce qui n'est pas le cas de la Polynésie française. En effet, le compte courant polynésien est réduit à $0,2 \%$ du PIB, grâce aux revenus nets du tourisme et des transports (en particulier, les passagers d'Air Tahiti Nui), et surtout aux transferts nets de l'Etat à la hauteur de $24 \%$ du PIB. Ces donations de l'Etat sont donc cruciales pour l'équilibre de la balance courante, mais aussi pour le maintien de la parité entre le franc CFP et l'euro. Pour résumer, la quasi-totalité du déficit de la balance des biens et services privés (c'est-à-dire la différence entre les importations et les exportations) de la Polynésie française est financé par les transferts publics de l'Etat. Plus précisément, les dépenses nettes des armées françaises en Polynésie française représentent 2,4\% du PIB, les rémunérations indexées des fonctionnaires de l'Etat mutés en Polynésie française sont égales à 10,2\% du PIB et les autres transferts des administrations publiques, dont les retraites indexées des fonctionnaires de l'Etat en Polynésie française, atteignent 11,6\% du PIB en 2005. Par conséquent, la métropole est responsable de plus de la moitié des transactions du compte courant, comme le confirme la deuxième partie du tableau, même si les exportations et importations de biens et de services privés de métropole ne représentent qu'un plus faible pourcentage.

BALANCE DES PAIEMENTS DE LA POLYNESIE FRANCAISE EN 2005

\begin{tabular}{|l|c|c|c|c|c|c|}
\hline \multicolumn{1}{|c|}{ Source: IEOM } & \multicolumn{3}{c|}{ avec le monde } & \multicolumn{3}{c|}{ avec la métropole } \\
\hline (En milliards de francs CFP) & Crédits & Débits & Soldes & Crédits & Débits & Soldes \\
\hline COMPTE COURANT & $\mathbf{2 5 1 , 7}$ & $\mathbf{2 5 0 , 9}$ & $+\mathbf{0 , 9}$ & $\mathbf{1 8 1 , 2}$ & $\mathbf{1 2 8 , 6}$ & $+\mathbf{5 2 , 6}$ \\
\hline $\begin{array}{l}\text { Balance des biens (= balance } \\
\text { commerciale) }\end{array}$ & $\mathbf{2 0 , 3}$ & $\mathbf{1 5 2 , 9}$ & $\mathbf{- 1 3 2 , 5}$ & $\mathbf{2 , 8}$ & $\mathbf{5 6 , 3}$ & $\mathbf{- 5 3 , 5}$ \\
\hline Balance des services & $\mathbf{1 0 2 , 9}$ & $\mathbf{6 9 , 8}$ & $+\mathbf{3 3 , 1}$ & $\mathbf{5 2 , 8}$ & $\mathbf{4 7 , 4}$ & $+\mathbf{5 , 4}$ \\
\hline Transports maritimes et aériens & 23,6 & 19,5 & $+4,1$ & 14,4 & 12,5 & $+1,9$ \\
\hline
\end{tabular}

32 André Cartapanis, Vincent Dropsy et Sophie Mametz "The Asian Currency Crises: Vulnerability, Contagion or Unsustainability?" (2002) 10 Review of International Economics 79-91. 


\begin{tabular}{|l|c|c|c|c|c|c|}
\hline Voyages & 50,0 & 29,0 & $+21,0$ & 13,5 & 20,2 & $-6,8$ \\
\hline Autres services privés & 17,3 & 21,2 & $-3,9$ & 13,0 & 14,6 & $-1,6$ \\
\hline $\begin{array}{l}\text { Services des administrations } \\
\text { publiques (dépenses des Armées) }\end{array}$ & 11,9 & 0,1 & $+11,8$ & 11,9 & 0,1 & $+11,8$ \\
\hline Balance des revenus & $\mathbf{5 6 , 4}$ & $\mathbf{6 , 4}$ & $+\mathbf{5 0 , 0}$ & $\mathbf{5 5 , 8}$ & $\mathbf{6 , 2}$ & $+\mathbf{4 9 , 6}$ \\
\hline $\begin{array}{l}\text { Rémunération des salariés } \\
\text { (fonctionnaires d'Etat) }\end{array}$ & 52,4 & 1,4 & $+51,0$ & 52,2 & 1,2 & $+51,0$ \\
\hline Revenus des investissements & 4,0 & 5,0 & $-1,0$ & 3,6 & 4,9 & $-1,3$ \\
\hline $\begin{array}{l}\text { Balance des transferts } \\
\text { courants }\end{array}$ & $\mathbf{7 2 , 1}$ & $\mathbf{2 1 , 8}$ & $+\mathbf{5 0 , 3}$ & $\mathbf{6 9 , 8}$ & $\mathbf{1 8 , 7}$ & $+\mathbf{5 1 , 1}$ \\
\hline $\begin{array}{l}\text { Secteur des administrations } \\
\text { publiques (y compris retraites de } \\
\text { fonctionnaires) }\end{array}$ & 64,2 & 6,2 & $+58,0$ & 64,1 & 5,8 & $+58,3$ \\
\hline $\begin{array}{l}\text { Autres transferts } \\
\text { COMPTE FINANCIER } \\
\text { (y compris compte de capital) }\end{array}$ & $\mathbf{3 7 8 , 6}$ & $\mathbf{3 8 1 , 8}$ & $\mathbf{- 3 , 2}$ & $\mathbf{3 7 1 , 7}$ & $\mathbf{3 7 0 , 9}$ & $\mathbf{0 , 8}$ \\
\hline $\begin{array}{l}\text { ERREURS ET OMISSIONS } \\
\text { PAIEMENTS }\end{array}$ & $\mathbf{2 , 4}$ & $\mathbf{0 , 0}$ & $\mathbf{2 , 4}$ & $\mathbf{0 , 0}$ & $\mathbf{5 3 , 4}$ & $\mathbf{- 5 3 , 4}$ \\
\hline $\begin{array}{l}\text { TOTAL BALANCE DES } \\
\text { (632,7 }\end{array}$ & $\mathbf{6 3 2 , 7}$ & $\mathbf{0 , 0}$ & $\mathbf{5 5 2 , 9}$ & $\mathbf{5 5 2 , 9}$ & $\mathbf{0 , 0}$ \\
\hline
\end{tabular}

Sur ce point, il est utile de faire une rapide comparaison avec la Nouvelle Calédonie, qui est également concernée par le passage à l'euro. Comme en Polynésie française, la balance des biens et des services privés est en déficit permanent, et les transferts de l'Etat financent la grande partie de ce déficit (si ce n'est même plus, comme en 2004), à hauteur d'environ $21 \%$ du PIB. Cependant, il existe une différence notable: la principale source de revenus extérieurs n'est pas le tourisme, mais le nickel. En effet, les exportations de ce produit minier représentent en moyenne $90 \%$ des exportations de biens, environ $60 \%$ des exportations de biens et services privés, et $25 \%$ du PIB calédonien. Les revenus nets du tourisme sont biens positifs, mais ils deviennent presque nuls si on y inclut les recettes nettes négatives des transports aériens (en particulier, les passagers d'Air France). En tout cas, la métropole reste de loin le premier partenaire commercial de la Nouvelle 
Calédonie, ${ }^{33}$ avec environ $30 \%$ des échanges de biens, 55\% des transactions du compte courant, et plus de $90 \%$ de transactions du compte financier en 2004. C'est pourquoi on peut également conclure que le choix d'une union monétaire avec la zone euro (c'est-à-dire le remplacement du franc CFP par l'euro) est optimal pour la Nouvelle Calédonie, ainsi que pour Wallis et Futuna, ${ }^{34}$ dont la dépendance économique envers les transferts de l'Etat est encore plus forte.

En ce qui concerne la Polynésie française, la situation de déficit chronique n'est pas nouvelle, puisqu'elle perdure depuis les années soixante. En effet, la balance des biens civils était en léger surplus en 1960, avant de plonger dans un déficit record d'environ 60\% du PIB en 1966 lors de la construction de l'aéroport de Faa'a et du CEP pour les essais nucléaires. La même année, le déficit de la balance des biens et services (y compris militaires) a atteint $82 \%$ du PIB, pour ensuite baisser graduellement jusqu'à environ $35 \%$ du PIB en moyenne dans les années soixante-dix, $27 \%$ du PIB dans les années quatre-vingt, 19\% du PIB dans les années quatre-vingt-dix. Il faut noter que ces chiffres sont des estimations basées sur diverses sources de données qui ne sont pas nécessairement homogènes, mais elles fournissent une idée de la tendance. En tout cas, on observe une forte corrélation de $86 \%$ entre le déficit de la balance des biens et services privés (c'est-à-dire en excluant les transferts publics) et les transferts nets de l'Etat en Polynésie française depuis 1959 et une corrélation toujours élevée de 75\% depuis 1970, après que le choc initial du CEP se soit dissipé. L'économie polynésienne (tout comme l'économie calédonienne) est également très dépendante des transferts de l'Etat pour deux raisons.

Premièrement, ces donations fournissent environ $30 \%$ du revenu local polynésien depuis plus de trente ans, et elles permettent de financer une très grande partie des importations, qui sont maintes fois supérieures aux exportations (y compris le tourisme). En effet, les recettes d'exportations de biens civils couvrent moins de $20 \%$ des dépenses d'importations de biens civils depuis maintenant quarante ans et a même presque chuté de moitié depuis 1999 (11\% en 2005). Par contre, en incluant les biens et services privés, ce taux de couverture a cru d'environ $10 \%$ jusqu'à une moyenne de $50 \%$ ces dernières années. Pour une fois, tous les économistes et politiciens s'accordent pour conclure qu'il faut continuer à réduire cette dépendance ${ }^{35}$ vis-à-vis de l'Etat, en développant le tourisme qui est la principale source de revenus privés de l'extérieur, mais qui ne représente aujourd'hui que $5 \%$ $\mathrm{du}$ PIB en termes nets (c'est-à-dire en soustrayant les dépenses des polynésiens voyageant à l'étranger, des revenus provenant des touristes venant visiter la Polynésie française).

Deuxièmement, l'Etat garantit la convertibilité illimitée et la parité du franc CFP par rapport à l'euro. Sans les transferts public métropolitains, le compte de l'IEOM auprès du Trésor public

33 Institut d'Emission d'Outre-Mer (2006) "La Nouvelle Calédonie en 2005", Rapport Annuel.

34 Institut d'Emission d'Outre-Mer (2006) "Wallis et Futuna en 2005", Rapport Annuel.

35 Montet, Christian (2005) "Pour un Taui Economique (2): La Politique Structurelle", Tahiti Pacifique n¹75, $32-33$. 
deviendrait chaque année déficitaire de 30\% du PIB polynésiens, soit environ 1,2 milliards d'euros, et on peut se demander pour combien de temps la parité franc CFP/euro serait défendue à ce prix-là. Dans ces conditions, si le taux de change du franc CFP par rapport à l'euro était soudainement livré aux forces des marchés de change, la logique économique veut que le franc CFP se déprécie très fortement pour renforcer la compétitivité des exportations polynésiennes et réduire les importations qui deviendraient beaucoup plus chères qu'au taux de change actuel. Cependant, la valeur des exportations de biens et de services privés étant seulement la moitié des valeurs des importations de biens et de services privés, il est fort probable que l'effet positif sur ces exportations soit moindre que l'effet inflationniste et dépressif sur les importations. Mes estimations économétriques laissent penser qu'une dépréciation de $10 \%$ augmenterait la valeur des exportations de biens et de services (dont le tourisme) par presque $20 \%$, mais n'aurait que peu d'effets sur la valeur des importations. Pour doubler ces exportations, il faudrait donc une dévaluation d'environ 50\% (comme celle du franc CFA en 1994), qui aurait un effet fortement inflationniste sur les prix (et donc dépressionnaire sur l'économie locale), puisque l'indice des prix à la consommation en Polynésie française est composé en moyenne de $40 \%$ de produits importés depuis une trentaine d'années. Par contre, il aurait été possible de neutraliser l'effet inflationniste d'une dévaluation d'environ 30\% en éliminant les taxes à l'importation (du même niveau) tout en gardant les transferts de l'Etat constants et donc les salaires des fonctionnaires en francs CFP, comme l'avait judicieusement proposé le professeur Poirine ${ }^{36}$ un éminent expert de l'économie polynésienne. ${ }^{37}$ Mais cette proposition a soulevé des oppositions politiques et quelques années plus tard (en 1997), une grande partie des taxes à l'importation a été remplacée par des taxes sur la valeur ajoutée (TVA) locale, ce qui rend cette proposition caduque aujourd'hui.

Si une dévaluation du franc CFP est pratiquement hors de question, il n'en demeure pas moins que le franc CFP est surévalué, selon toute logique économique. Il existe bien des théories économiques sur la valeur d'équilibre du taux de change (parité de pouvoir d'achat, modèle de Balassa-Samuelson, approche monétaire, etc...), mais des difficultés techniques bien connues des spécialistes rendent ces théories difficilement applicables pour estimer le niveau de surévaluation d'une monnaie, et en particulier du franc CFP. A titre d'exemple au début du mois de janvier 2006, un Big Mac ${ }^{38}$ coûtait 470 francs CFP à Tahiti et 3,10 dollars US aux Etats-Unis, alors que le taux de change entre les deux monnaies était d'environ 100 francs CFP par dollar US. Le Big Mac américain valait donc 310 francs CFP, soit 34\% de moins que le Big Mac tahitien. Vice versa, le prix d'un Big Mac tahitien était de 4,70 dollars US, soit 52\% plus élevé qu'un Big Mac américain.

36 Bernard Poirine (1994) "Le Franc Pacifique: un avenir à Tahiti ?" Tahiti Pacifique, n44, 15-21.

37 Bernard Poirine Tahiti, stratégie pour l'après-nucléaire (2 $2^{\mathrm{ème}}$ éd, L'Harmattan, Paris, 1996).

38 The Economist (2006, may 25) "Economics focus: McCurrencies (Happy 20th birthday to our Big Mac index)". 
En tout cas, cette surévaluation de la monnaie polynésienne est un frein aux exportations, et en particulier au tourisme ${ }^{39}$. Bien que plus de $89 \%$ des touristes soient satisfaits au point qu'ils recommanderaient cette destination à leur entourage, le coût de la vie très élevé est de loin la première cause d'insatisfaction parmi eux ${ }^{40}$. On retrouve d'ailleurs dans d'autres enquêtes le même sentiment de cherté de la vie parmi la population locale. Ce coût de la vie très élevé et le manque de compétitivité internationale qui s'ensuit, peuvent continuer à perdurer tant que l'Etat poursuit son financement du déficit externe privé de la Polynésie française par l'intermédiaire de ses importants transferts publics annuels. Substituer l'euro au franc CFP ne ferait qu'entériner cette condition sine qua non en éliminant la possibilité d'une dévaluation, même si cette dernière n'est pas une solution avantageuse dans les conditions présentes. La problématique macroéconomique n'est donc pas le choix entre le régime actuel de change fixe par rapport à l'euro et l'adoption de la monnaie européenne, mais bien la forte dépendance de l'économie polynésienne vis-à-vis des contributions de l'Etat. On peut donc se demander si l'éventuel passage à l'euro ne peut pas être considéré comme une stratégie économique ou politique provenant de la théorie des jeux. En supprimant toute éventualité de dévaluation, les décisionnaires réduiraient leur champs d'action à une seule solution économique pour ne pas sombrer dans une crise, à savoir le maintien, voire même l'augmentation de la $\left\langle\right.$ rente» ${ }^{41}$ versée par l'Etat, en attendant que l'économie polynésienne ne développe un niveau de ressources propres suffisant pour compenser cette rente annuelle de l'ordre de $30 \%$ de son PIB.

On peut néanmoins conclure que, ceteris paribus, la Polynésie française semble remplir les conditions microéconomiques et macroéconomiques pour qu'un changement de régime de change vers une union monétaire avec la zone euro lui apporte des avantages supérieurs aux inconvénients, à condition de préparer la population et de prendre des précautions pour éviter des dérapages de prix lors de la transition vers l'euro. En particulier, on pourrait observer une accélération de la convergence entre les prix à la consommation en Polynésie française et en métropole, phénomène déjà observé en tendance depuis 1985.

\section{CONCLUSIONS}

La Polynésie française a bénéficié depuis presque soixante ans d'un régime de change parfaitement stable, grâce à un statut juridique et monétaire particulier, à savoir la garantie de la part des autorités métropolitaines, d'une convertibilité illimitée de sa monnaie, le franc CFP, et du maintien de sa parité fixe par rapport à l'euro (ou au franc français avant 2002). Le remplacement du franc CFP par l'euro, souhaité par l'Assemblée de la Polynésie française dans une résolution en

39 Institut Statistique de la Polynésie Française " L'impact économique du tourisme international sur l'économie polynésienne" (2006) Points Forts de la Polynésie Française, n5.

40 Institut Statistique de la Polynésie Française " Destination Polynésie française: impression des touristes internationaux" (2005) Points Forts de la Polynésie Française, n4.

41 Bernard Poirine (1993) "Le développement par la rente dans les petites économies insulaires" (1993) 44 Revue Economique n6, 1169-1199. 
janvier 2006, ne semble pas présenter de problèmes majeurs au niveau institutionnel européen, à condition que les deux autres collectivités d'outre-mer, la Nouvelle Calédonie, et Wallis et Futuna, soient également d'accord.

L'analyse des critères de zone monétaire optimale montre que la Polynésie française satisfait la plupart des conditions d'adhésion à l'Union Monétaire Européenne, et en particulier, que les importants transferts de l'Etat sont un facteur crucial d'intégration économique et financière avec la métropole. C'est pourquoi l'euro plutôt que le dollar US est le choix optimal pour se substituer au franc CFP. De plus, ce rattachement à la zone euro apporterait des avantages microéconomiques à l'économie polynésienne, telles que: (i) l'élimination du risque de dévaluation, qui ferait baisser les taux d'intérêts; (ii) la facilitation des échanges commerciaux, qui bénéficierait aux exportations; (iii) l'amélioration de la transparence des prix relatifs à l'étranger, qui induirait une baisse des prix. Il faudrait néanmoins prendre garde à préparer la population locale et à éviter des dérapages de prix lors de la période de transition. Les risques macroéconomiques ne proviendraient pas d'un passage à l'Euro, mais plutôt de la dépendance importante envers les transferts de l'Etat, qui ont permis à la Polynésie française de financer son large déficit extérieur privé depuis plus de quarante ans, et d'éviter une dévaluation pratiquement inéluctable en l'absence de ces transferts. La suppression du franc CFP au profit de l'euro éliminerait ainsi toute possibilité de dévaluation et renforcerait l'engagement de l'Etat, soit à aider la Polynésie française à se développer économiquement pour réduire sa dépendance financière, soit à augmenter ces transferts pour éviter toute crises économique.

La conclusion de cette analyse est donc qu'une union monétaire avec la zone euro est le choix optimal de régime de change dans le contexte actuel de la Polynésie française, mais qu'il reste nécessaire de réduire la dépendance de l'économie polynésienne vis-à-vis des transferts financiers de l'Etat. 
